\title{
Research on High-rise Building Fire Early Warning System Based on Multidimensional Data Fusion
}

\author{
Kong Shengli ${ }^{1}$, Fang Xinmei ${ }^{1}$, Yang Pengfei ${ }^{1}$, Yang Zhen ${ }^{1}$ and Wang $\mathrm{Wei}^{2}{ }^{2 *}$ \\ ${ }^{1}$ School of Urban Construction and Safety Engineering, Shanghai Institute of Technology, Shanghai 201418, China \\ ${ }^{2}$ Shanghai Fire Science and Technology Research Institute of MEM, Shanghai 200032, China
}

\begin{abstract}
With the rapid development of urban construction and the increasing number of high-rise buildings, many problems have arisen, one of which is fire. High-rise building fires are developing rapidly, and it is difficult to extinguish them, which causes great losses and seriously threatens people's safety in production and life. Early warning of high-rise building fire is an important means to prevent fire. In this paper, aiming at high-rise building fires, the causes of high-rise building fires in 2019 are analyzed statistically, and the causes of high-rise building fires are summarized. An intelligent early warning system of high-rise building fire based on multi-dimensional data fusion is proposed. Based on the actual forecast data, the early warning system based on multi-dimensional data fusion is analyzed through Matlab simulation, thus verifying the feasibility and reliability of the established fire early warning system. The research results have certain guiding significance for high-rise building fire warning.
\end{abstract}

\section{Introduction}

According to statistics, there are about 600,000 high-rise buildings (including public buildings and residential buildings) in China, among which about 6,000 are super high-rise buildings (with a height above $100 \mathrm{~m}$ ). In recent 10 years, there have been about 30,000 high-rise building fires, with 474 deaths [1]. It shows that in the current social development, the challenges faced by fire safety management should not be underestimated.

In the current technological and social environment, high-rise buildings are characterized by large number of people, complex structure and complicated electrical equipment. Fire-fighting equipment in China is difficult to meet its fire-fighting needs. Once a fire occurs, it is very difficult to save and evacuate people [2-3]. Now, with the rise of various Internet and Internet of Things technologies, we can use artificial intelligence, Internet interaction, Internet of Things control and other means for data and intelligent management [4]. Therefore, when a fire breaks out in a high-rise building, managers can control firefighting facilities and organize personnel evacuation scientifically, which is of great help to improve the fire monitoring and management of high-rise buildings, improve the speed of emergency response and reduce casualties.

\section{Fire causes of high rise buildings}

The author counts the number of high-rise buildings in China from 2016 to 2020, and the number of accidents is shown in Figure 1.

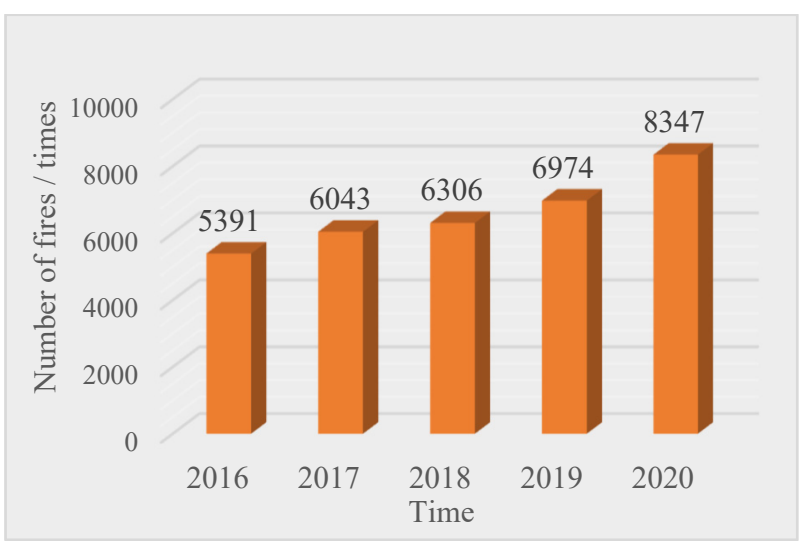

Figure 1. Number of high-rise building fires in China from 2016 to 2020

It can be seen from the figure that the number of fires in high-rise buildings is increasing year by year, so it is necessary to summarize and analyze the causes of fires. According to the statistics of the number of fires reported by the Fire Department of the Ministry of Public Security in 2019, a total of 6,794 fires occurred in high-rise buildings throughout the year. The causes of fires in highrise buildings were statistically analyzed, and the results are shown in Figure 2. 


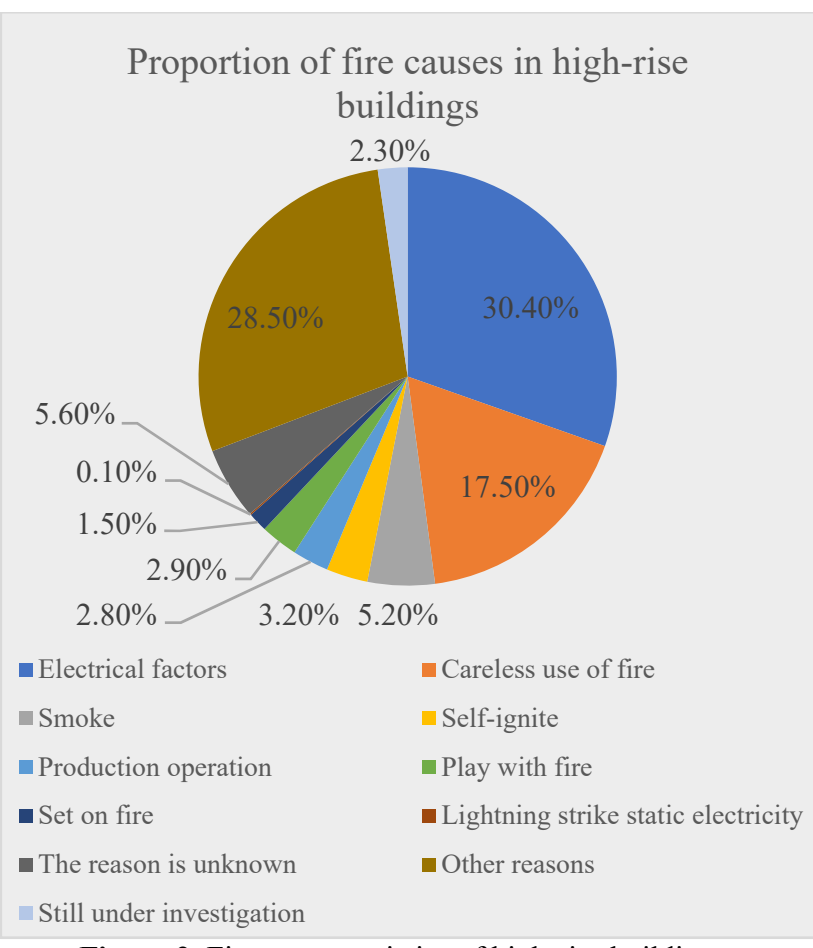

Figure 2. Fire cause statistics of high-rise buildings

As can be seen from the figure that there are various causes of high-rise building fires, among which electrical factors account for the largest proportion, reaching $30.40 \%$, with about 2,065 fires, which have brought serious threats to people's lives and property. Therefore, how to reduce or restrain the fire from the source has become an urgent problem to be solved.

As an important means to prevent fire, the fire early warning system of high-rise buildings needs to be built first.

\section{Construction of intelligent early warning system for high-rise building fire}

\subsection{Software design of the system}

\subsubsection{Structural framework design of multidimensional data fusion}

Firstly, the database is established, and the format handler for data uploading is directly added to the modules of each node and sensor [5]. According to the requirements, two databases are established, one database is used to store the data that can be used directly, including time, user location, temperature, humidity, wind speed, carbon monoxide content and water pipe pressure. In the second database, we save some miscellaneous data, specifically the users under the access program, their telephone numbers and signal connection strength, and the numbers of property and fire alarm.

After the establishment of related database theory, the framework of related multidimensional data is established. The data are divided into three dimensions. The first dimension is time and sensor position, which are used to reflect the position and time; the second dimension is temperature, humidity, carbon monoxide content and wind speed, which are used to judge the probability of fire; and the third dimension is water pipe pressure, which is used to call fire prevention/extinguishing facilities in combination with the first two data. Its specific functions are as follows: (1)The first dimension data can interact with the data uploaded by PC/APP terminal, and send relevant fire warning by comparing the terminal position. (2) The second-dimensional data can be operated in the server to calculate the fire occurrence probability. (3)Onedimensional and two-dimensional correlation processing can directly reflect the specific location where fire may occur. (4) The combination of the three dimensions can complete the early warning of fire possibility at each location. Once a fire is found, fire prevention facilities can be called and fire warning can be carried out, and fire evacuation information can be released.

\subsubsection{Algorithm modeling}

The values detected by sensors are very different from the data such as dimension. If they are used directly, the weight of the data will be reflected at the beginning [6], and there will be data errors. Therefore, it is necessary to normalize the data to eliminate the differences in size and dimension of various data. The main application formula is as follows:

$$
X_{i}^{\prime}=\frac{\left(X_{i}-X_{\min }\right)}{\left(X_{\max }-X_{\min }\right)}
$$

Among them, $\mathrm{X}_{\mathrm{i}}$ is the value of a sensor's output signal; $\mathrm{X}_{\min }$ and $\mathrm{X}_{\max }$ represent the minimum and maximum value of the sensor's output respectively; $\mathrm{X}_{\mathrm{i}}^{\prime}$ is the data after data normalization.

In this paper, BP neural network [7] is used to train and learn the detected humidity, temperature and $\mathrm{CO}_{2}$ concentration signals, and the corresponding network weight parameters are obtained. Four nodes are selected for the input layer, which correspond to humidity, temperature, $\mathrm{O}_{2}$ and $\mathrm{CO}_{2}$ concentration signals respectively. The hidden layer sets eight nodes according to experiment and experience, and the setting of this node is not based on concrete basis, but on the empirical results obtained by constant attempts; The three nodes in the output layer correspond to the open flame probability, smoldering probability and fire-free probability respectively.

The neural network training sample set is $\mathrm{X}^{\prime}=\left[\mathrm{X}_{1}{ }^{\prime}\right.$, $\left.\mathrm{X}_{2}{ }^{\prime}, \cdots, \mathrm{X}_{\mathrm{N}}{ }^{\prime}\right]$, and each sample $\mathrm{X}_{\mathrm{m}}{ }^{\prime}=\left[\mathrm{X}_{\mathrm{m} 1}{ }^{\prime}, \mathrm{X}_{\mathrm{m} 2}{ }^{\prime}, \cdots, \mathrm{X}_{\mathrm{mI}}{ }^{\prime}\right]$ in the training sample set is an I-dimensional vector with $\mathrm{m}=1,2, \cdots, \mathrm{N}$. The predicted output vector of the network is $\mathrm{O}_{\mathrm{m}}=\left[\mathrm{O}_{\mathrm{m} 1}, \mathrm{O}_{\mathrm{m} 2}, \cdots, \mathrm{O}_{\mathrm{mk}}\right] \mathrm{T}$, the expected output vector is $\mathrm{Y}_{\mathrm{m}}=\left[\mathrm{Y}_{\mathrm{m} 1}, \mathrm{Y}_{\mathrm{m} 2}, \cdots, \mathrm{Y}_{\mathrm{mk}}\right] \mathrm{T}$, the hidden layer contains $\mathrm{J}$ neurons, and the threshold corresponding to any hidden layer neuron $\mathrm{J}$ is set as $\theta_{j}$; The output layer is represented by $\mathrm{K}$, and the threshold corresponding to any output $\mathrm{k}$ is set as $\theta_{k}$; The connection weight between the input layer and the hidden layer is set to $\mathrm{W}_{\mathrm{ij}}$ (the weight between the ith input layer neuron and the jth hidden layer neuron); The connection weight of the hidden layer and 
the output layer is set as $\mathrm{W}_{\mathrm{jk}}$; The input and output of neurons are set to $\mu$ and $v$ respectively. The number of training iterations is $\mathrm{n}$. After initializing weights and thresholds, training and learning can be carried out, and the network learning signal is transmitted forward as follows. $\mu_{j}^{J}$ and $v_{j}^{J}$ are the input and output of the Jth neuron in the hidden layer, respectively. As shown in formula (2) and (3):

$$
\begin{gathered}
\mu_{j}^{J}=\sum_{j}^{J}\left(W_{i j} X_{m i}^{\prime}-\theta_{j}\right) \\
v_{j}^{J}=f\left(\mu_{j}^{J}\right)
\end{gathered}
$$

$\mu_{k}^{K}$ and $\mathrm{O}_{\mathrm{mk}}$ are the input and output of the kth neuron in the output layer, respectively, as shown in formula (4) and (5):

$$
\begin{aligned}
& \mu_{k}^{K}=\sum_{j}^{J}\left(W_{j k} v_{j}^{J}-\theta_{k}\right) \\
& O_{m k}=v_{k}^{K}=f\left(\mu_{k}^{K}\right)
\end{aligned}
$$

Output error $\mathrm{e}_{\mathrm{mk}}$ of $\mathrm{kth}$ neuron in output layer is shown in formula (6):

$$
e_{m k}(n)=Y_{m k}(n)-O_{m k}(n)
$$

Total system error E, as shown in formula (7):

$$
E(n)=\frac{1}{2} \sum_{K-1}^{K}\left(e_{m k}(n)\right)^{2}
$$

\subsection{Hardware design of the system}

This paper divides the selection of hardware into three parts, namely, the selection of sensor, the selection of processor and the theoretical design of hardware setting mode. In the selection of sensors, attention should be paid to minimize the influence of accuracy on prediction results, improve the accuracy of prediction results, and reduce costs as much as possible while ensuring accuracy. When selecting the processor, it can meet the data processing speed and reduce the actual construction cost as much as possible. The way of hardware setting is very important, because all measures and facilities to ensure safety must first meet the intrinsic safety, so how to set, the measures and methods to set, and the methods to deal with the normal operation of hardware in case of fire become extremely important [8].

At the same time, the choice of transmission mode is also very important. Choosing the appropriate transmission mode can greatly improve the overall data accuracy and efficiency. At present, the mainstream transmission modes in the market are Bluetooth, WiFi and ZigBee [9]. WiFi has the fastest data transmission speed and the highest transmission efficiency, and its nodes are relatively many. However, the disadvantage of $\mathrm{WiFi}$ is that it needs a fixed and continuous power supply for continuous power supply to ensure its normal operation, which leads to its possible fire [10]. Compared with WiFi, purple bee has more network nodes, lower cost and power consumption, but lower data transmission rate; Bluetooth is suitable for short-distance transmission [11]. Although the power consumption is the smallest, its advantages are not so obvious compared with the other two [12]. However, this paper needs a transmission mode with fast transmission speed, long transmission distance, strong signal penetration ability and convenient networking, so we choose purple bee transmission.

\subsubsection{Selection of sensors}

In this paper, we do not choose a specific type of sensor, but choose the built-in chip of the sensor module to choose the sensor, which can make its practical possibility higher, because there are many kinds of sensor products in the market now, and if we start with a single specific type of product, it will lead to lower selectivity, which is not conducive to upgrading and replacement in actual use [13]. Considering the requirements of practical application, SHT-75 temperature sensor with low power consumption, fast response and high stability is selected. Its temperature detection range is $-40 \sim 123.8^{\circ} \mathrm{C}$, the resolution of $\mathrm{HT}-75$ is 14 bits by default, and the detection accuracy is $0.3^{\circ} \mathrm{C}$. It communicates with wireless communication CC2530 controller through two-wire serial port. The MQ-7 sensor sensitive to $\mathrm{CO}$ gas is selected as its $\mathrm{CO}$ sensor. MQ -7 is a four-wire module (VCC, GND, D0, A0). Digital interface $\mathrm{D} 0$ outputs 1 (CO concentration is lower than the set threshold) and 0 (CO concentration is higher than the set threshold). Analog interface A0 output voltage increases with the increase of $\mathrm{CO}$ concentration.

\subsubsection{Selection of processors}

In this paper, the wireless communication module with the international standard communication frequency band of $2.4 \mathrm{GHz}$ is used, which has the characteristics of low-speed and small-area wireless transmission of information. The main components are microcontroller and RF chip. In this design, stm32f103, which is a small-speed processor produced by ARM, is used for micro-processing. CC2530 is used as communication chip, and there are power supply circuit, crystal oscillator circuit, serial port chip and external interface circuit in the whole single-channel design. The external interface circuit is mainly for the expansion of other functions and the renewal of system circuit.

At the same time, according to the actual demand, in the process of integration after selecting the processor, program it and put it into the protocol and format processing program

\subsubsection{Design of hardware setting mode}

When designing each node, according to the nature of ZigBee, we use tree topology to set the overall structure of each node. In theory, one node manages a kind of equipment, peers do not communicate with each other, and upper and lower nodes interact with each other. When uploading data, they are packaged and summarized level by level, and when descending data, they receive according to the data header [14]. The overall data structure mode and data transmission mode are shown in Figure 3. 


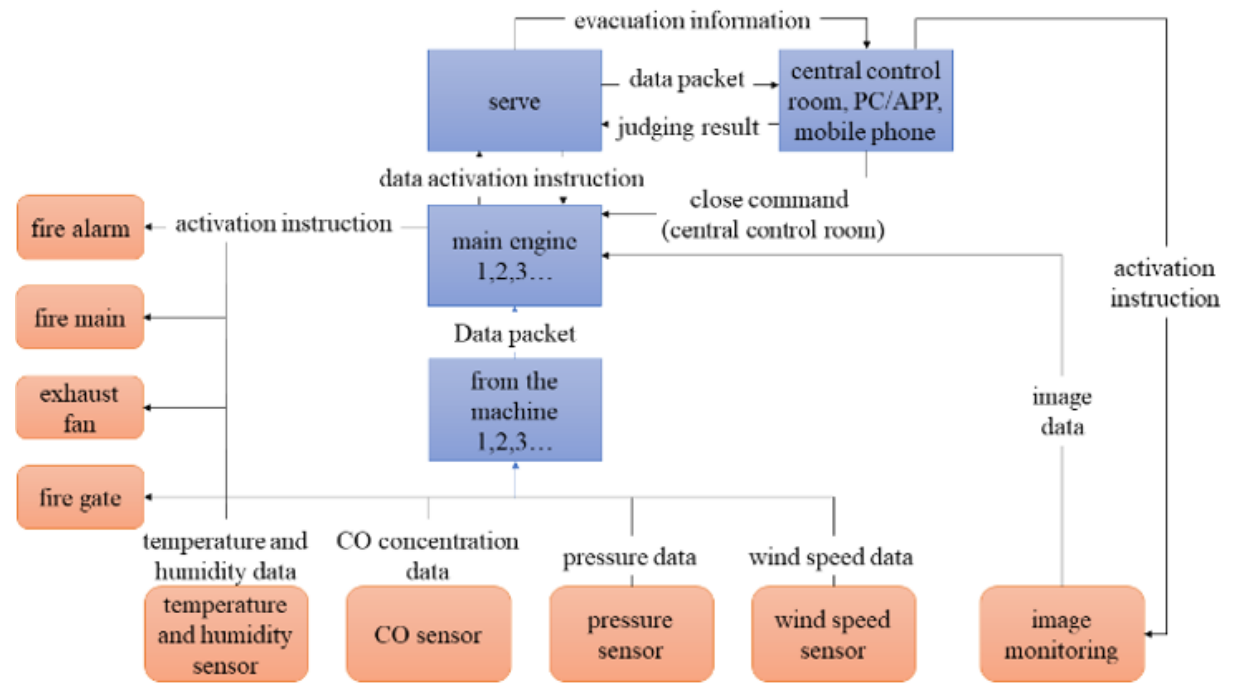

Figure 3. Hardware setup diagram

\section{Empirical analysis}

In order to verify the feasibility and effectiveness of the above-mentioned high-rise building fire warning system based on multidimensional data, it is analyzed and verified according to the simulated data. The data samples are shown in Table 1.

Table 1. Data sample

\begin{tabular}{|c|c|c|c|c|c|}
\hline grade & time $/ \mathrm{s}$ & $\begin{array}{c}\mathrm{CO} / \\
\mathrm{kg} / \mathrm{m}^{3}\end{array}$ & $\begin{array}{c}\mathrm{RH} / \\
\mathrm{kg} / \mathrm{m}^{3}\end{array}$ & $\begin{array}{c}\mathrm{O} 2 / \\
\mathrm{kg} / \mathrm{m}^{3}\end{array}$ & $\begin{array}{c}\mathrm{TEMP} / \\
\mathrm{kg} / \mathrm{m}^{3}\end{array}$ \\
\hline 1 & 0 & 0.000695338 & 0.006274379 & 0.26878595 & 24.999992 \\
\hline 2 & 151.54 & 0.000695335 & 0.006274357 & 0.26878501 & 25.000937 \\
\hline 3 & 302.62 & 0.000695335 & 0.006274356 & 0.26878499 & 25.001008 \\
\hline 4 & 450.05 & 0.000695337 & 0.006274374 & 0.26878573 & 25.000281 \\
\hline 5 & 601.58 & 0.000695338 & 0.006274383 & 0.26878611 & 24.999828 \\
\hline 6 & 749.01 & 0.000695337 & 0.006274375 & 0.26878579 & 25.000141 \\
\hline 7 & 900.09 & 0.000695337 & 0.006274374 & 0.26878574 & 25.000225 \\
\hline 8 & 1051.63 & 0.000695337 & 0.006274377 & 0.26878589 & 25.000063 \\
\hline 9 & 1199.06 & 0.000695337 & 0.006274377 & 0.26878586 & 25.000090 \\
\hline 10 & 1350.14 & 0.000695337 & 0.006274377 & 0.26878588 & 25.000073 \\
\hline 11 & 1501.22 & 0.000695338 & 0.006274380 & 0.26878599 & 24.999967 \\
\hline 12 & 1649.10 & 0.000695338 & 0.006274381 & 0.26878603 & 24.999917 \\
\hline 13 & 1800.18 & 0.000695338 & 0.006274381 & 0.26878604 & 24.999912 \\
\hline
\end{tabular}

According to practical experience, the probability of fire-free, smoldering and open fire are predicted, as shown in Figure 4. 


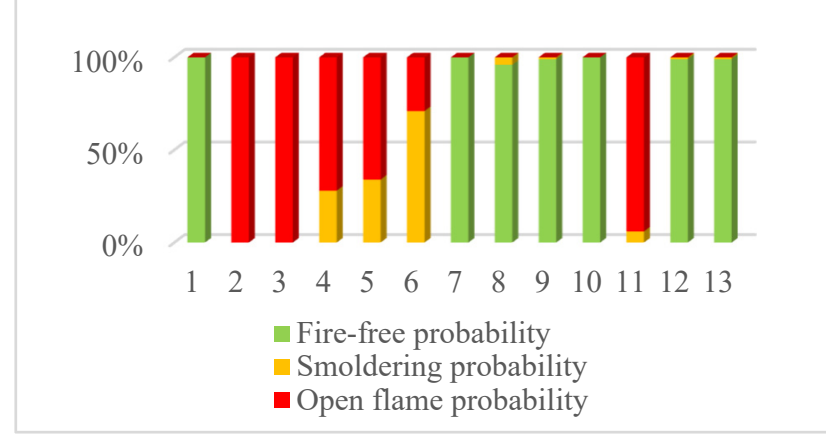

Figure 4. Predicted output probability 5.

In the intelligent algorithm, the output result is Figure

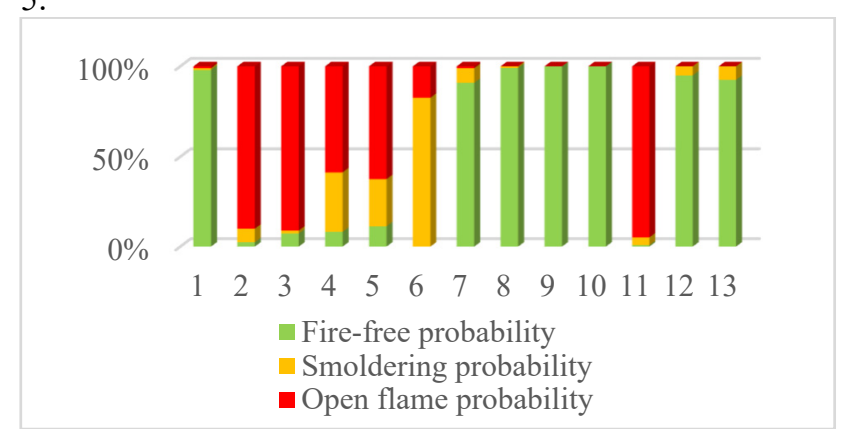

Figure 5. Output probability of intelligent algorithm

Comparing fig. 4 with fig. 5 , it can be concluded that the open flame probability output by the intelligent algorithm is basically consistent with the actual result.

\section{Conclusion}

In this paper, an intelligent fire early warning system for high-rise buildings based on multi-dimensional data fusion is proposed, and the feasibility and reliability of the established fire early warning system are verified by empirical analysis of common fire data. However, the algorithm has a training process, which may lead to inaccurate results and needs further improvement.

\section{Acknowledgements:}

Sponsored by National Science Foundation of China (51604174； 51706122) , Shanghai Rising-Star Program (20QB1401000), the Natural Science Foundation of Shanghai (19ZR1411500), the Science \& Technology Foundation of Guizhou Province ([2020]4Y055). "Chen Guang ” project supported by Shanghai Municipal Education Commission and Shanghai Education Development Foundation (17CG60)

\section{References}

1. Chao. Cheng, Xiaojia. Huang, Shuibo. Xie, Dongli. Wu, Wei. Jiang, Fire Science and Technology 37, 841(2018)

2. Xueliang. Liu, Guangdong Building Materials 27, 90(2011)

3. Yang. Yang, Fire Science and Technology 38,
1019(2019)

4. Zhenguo. Zhang, Fire Science and Technology 39, 1739(2020)

5. Sixiang. Zhang, Kai. Gan, Wei. Zhou, Transducer and Microsystem Technologies 40, 151(2021)

6. Sitong. Zeng, Xiaowei. Tong, Peihui. Shen, Journal of Hubei Polytechnic University 35, 23(2019)

7. Ping. Zhao, Qian. Xiong, Xin. Zhang, Guangchuan. Liu, Journal of Safety Science and Technology 16, 122(2020)

8. Lining. Zhang, Qi. Zhang, Jing. An, safety and environmental engineering 22, 140(2015)

9. Juan. Huang, Haodong. Cheng, Yongfeng. Li, Qince. Liang, Fire Science and Technology 38, 973(2019)

10. Jianqiang. He, Yao. Chen, Bianchi. Wang, Automation \& Instrumentation 34, 89(2019)

11. Cheng. Xie, Technology and Economic Guide 29, 25(2021)

12. Chaoran. Wang, Qiang. Liu, Shenyue. Wang, Changbo. Hou, Computer Measurement \& Control 25, 133(2017)

13. Dingbo. Zhang, Engineering and Technological Research 4, 110(2019)

14. Ming. Tang, Jing. Zheng, Wenting. Huang, Di. Wu, Hai. Huang, Chinese Journal of Sensors and Actuators 31, 312(2018) 